\title{
Extracellular matrix metalloproteinase inducer (CD147) and membrane type 1-matrix metalloproteinase are expressed on tissue macrophages in calcific aortic stenosis and induce transmigration in an artificial valve model
}

\author{
Nader Joghetaei, MD, ${ }^{\mathrm{a}}$ Payam Akhyari, MD, ${ }^{\mathrm{b}}$ Bernhard H. Rauch, MD, ${ }^{\mathrm{c}}$ Paul Cullen, MD, ${ }^{\mathrm{d}}$ \\ Artur Lichtenberg, MD, ${ }^{\mathrm{b}}$ Martina Rudelius, MD, ${ }^{\mathrm{e}}$ Jaroslav Pelisek, MD, ${ }^{\mathrm{f}}$ and Roland Schmidt, $\mathrm{MD}^{\mathrm{a}}$
}

\begin{abstract}
Objective: Matrix metalloproteinases participate in remodeling of extracellular matrix, which is central to the development of aortic stenosis. Synthesis of certain matrix metalloproteinases is induced by the glycoprotein extracellular matrix metalloproteinase inducer. We investigated whether extracellular matrix metalloproteinase inducer and membrane-type 1 matrix metalloproteinase are abundant in calcific aortic valve and their role in the pathogenesis of this condition.
\end{abstract}

\begin{abstract}
Methods: Sixteen patients who underwent surgery for aortic stenosis $(\mathrm{n}=12)$ or heart transplantation for ischemic cardiomyopathy $(n=4)$ were reviewed. Expression of extracellular matrix metalloproteinase inducer and membrane-type 1 matrix metalloproteinase proteins was assessed by Western blot $(n=4$ per group), immunohistochemistry for aortic stenosis $(\mathrm{n}=12)$ and ischemic cardiomyopathy $(\mathrm{n}=2)$, and in situ zymography $(\mathrm{n}=3$ per group). Functional relevance was investigated using an artificial valve model.
\end{abstract}

Results: Extracellular matrix metalloproteinase inducer and membrane-type 1 matrix metalloproteinase were abundant in all stenotic valves. Control valves did not stain for either protein. Double immunofluorescence colocalized extracellular matrix metalloproteinase inducer and membrane-type 1 matrix metalloproteinase to macrophages. On Western blotting, both proteins were more abundant in stenotic valves than in control valves. In situ zymography demonstrated greater gelatinolytic activity in stenotic valves than in control valves. Silencing of the extracellular matrix metalloproteinase inducer gene using small interfering RNA reduced migration of monocytes in an artificial valve model.

Conclusions: Extracellular matrix metalloproteinase inducer and membrane-type 1 matrix metalloproteinase were demonstrated on macrophages in stenotic aortic valves, into which extracellular matrix metalloproteinase inducer may promote monocyte immigration. The latter protein may therefore represent a potential target to reduce the development of aortic stenosis. (J Thorac Cardiovasc Surg 2011;142:191-8)

Aortic stenosis is the most common valvular heart disease in Western countries ${ }^{1}$ and the most common indication for aortic valve replacement. ${ }^{2} \mathrm{Up}$ to a few years ago, aortic stenosis was generally regarded as a mechanical problem to

\footnotetext{
From the Deutsches Herzzentrum und I. Medizinische Klinik, ${ }^{a}$ Klinikum rechts der Isar, Technische Universität München, München, Germany; Clinic for Cardiovascular Surgery, ${ }^{\mathrm{b}}$ Heinrich Heine University, Düsseldorf, Germany; Institut für Pharmakologie und Klinische Pharmakologie Heinrich Heine University, ${ }^{\mathrm{c}}$ Düsseldorf, Germany; Medizinisches Versorgungszentrum für Laboratoriumsmedizin Dr. Löer, Dr. Treder und Kollegen, ${ }^{\mathrm{d}}$ Münster, Germany; Institut für Pathologie, Klinikum rechts der Isar, Technische Universität München, München, Germany; and Klinik für Gefässchirurgie, ${ }^{f}$ Klinikum rechts der Isar, Technische Universität München, München, Germany.

This work was supported by a grant to Roland Schmidt from the German Heart Foundation/German Foundation of Heart Research (F06/07).

Disclosures: Authors have nothing to disclose with regard to commercial support.

Received for publication Dec 20, 2009; revisions received Sept 12, 2010; accepted for publication Sept 26, 2010; available ahead of print Dec 13, 2010.

Address for reprints: Roland Schmidt, MD, Deutsches Herzzentrum und 1. Medizinische Klinik, Klinikum rechts der Isar, Technische Universität München, Lazarettstr. 36, 80636 München, Germany (E-mail: rolschmidt@yahoo.de). $0022-5223 / \$ 36.00$

Copyright (C) 2011 by The American Association for Thoracic Surgery doi:10.1016/j.jtcvs.2010.09.051
}

be solved by replacement surgery when severe symptoms developed.

This view has changed recently. Calcific aortic stenosis is no longer regarded as an immutable degenerative disease, and research into measures aimed at slowing or even halting the process has gathered speed. ${ }^{3}$ Inflammatory mechanisms known to operate in atherosclerosis have been shown to operate in degenerative aortic stenosis, ${ }^{4}$ albeit with some differences $^{5}$ so that calcific aortic stenosis is increasingly regarded as an active inflammatory disease involving monocytes as the main cellular players.

Transmigration of monocytes into the subendothelium is strongly associated with matrix metalloproteinases (MMPs). Increased presence of MMP-2 and MMP-9 has been demonstrated in diseased tricuspid and bicuspid aortic valves. ${ }^{6}$ Inflammation has been shown to be strongly associated with calcium deposits in atherosclerosis and aortic valve stenosis. ${ }^{7-9}$ In aortic valve myofibroblasts, calcification mediated by the Wnt/LRP5/ß-catenin signaling cascade is activated by oxidized low-density lipoprotein and inhibited by statins. ${ }^{10}$ Further antiinflammatory 


$$
\begin{aligned}
& \text { Abbreviations and Acronyms } \\
& \begin{aligned}
\text { ECM }= & \text { extracellular matrix } \\
\text { EMMPRIN }= & \text { extracellular matrix } \\
& \text { metalloproteinase inducer } \\
\text { MMP }= & \text { matrix metalloproteinase } \\
\text { MT1-MMP }= & \text { membrane type 1-matrix } \\
& \text { metalloproteinase } \\
\text { NF } \kappa-\mathrm{B}= & \text { nuclear factor kappa-B } \\
\text { siRNA }= & \text { small interfering RNA } \\
\text { SMC } & \text { smooth muscle cell } \\
\text { TNF- } \alpha= & \text { tumor necrosis factor alpha }
\end{aligned}
\end{aligned}
$$

effects of statins include a decrease in the expression of proinflammatory interleukin- $1 \mathrm{~b}$ in endothelial cells ${ }^{11}$ and tumor necrosis factor (TNF)- $\alpha$ in macrophages, ${ }^{12}$ and reduced proliferation of smooth muscle cells (SMCs). ${ }^{13}$ Statins have also been shown to prevent calcification of vascular SMCs. ${ }^{14}$ In clinical trials, however, statins have thus far failed to halt or regress aortic valve stenosis. ${ }^{15}$ Therefore, a more nuanced view on the inflammatory process is warranted.

Calcification is governed by complex interactions between the cellular components and the extracellular matrix (ECM) of the aortic valve, with pathologic remodeling resulting from an imbalance between synthesis and breakdown of ECM.

MMPs are known to play an important role in atherosclerotic plaque instability. ${ }^{16}$ De novo synthesis of certain metalloproteinases, such as MT1-MMP, MMP-2, and MMP-9, is induced by the immunoglobulin-like glycoprotein extracellular matrix metalloproteinase inducer (EMMPRIN) (also known as basigin or CD147). ${ }^{17} \mathrm{We}$ previously showed that EMMPRIN is up-regulated on monocytes of patients with acute myocardial infarction. ${ }^{18}$ Moreover, atherogenic stimuli were shown to up-regulate EMMPRIN protein expression on monocytes, which in turn stimulated MMP-2 protein expression in SMCs. ${ }^{19}$

The present study investigated whether EMMPRIN is abundant in the aortic valve, and if so, whether it may contribute to the pathogenesis of calcific aortic stenosis.

\section{MATERIALS AND METHODS Patients}

Aortic valve fragments were collected from 16 patients undergoing surgery for aortic stenosis $(\mathrm{n}=12)$ or heart transplantation for ischemic cardiomyopathy $(\mathrm{n}=4)$. Valve fragments were used for histologic studies ( $\mathrm{n}=12$ for aortic stenosis and $\mathrm{n}=2$ for ischemic cardiomyopathy), and when available, for Western blot analysis ( $n=4$ for either group) and in situ gelatin zymography ( $\mathrm{n}=3$ for either group).

The ethics committee approved the study protocol, and written, informed consent was obtained from patients.

\section{Cells}

Human MonoMac6 cells were cultured in VLE-RPMI-1640 (Biochrom, Cambridge, UK) containing 10\% low-tox fetal calf serum (Clonetics, San Diego, Calif). MonoMac6 represents monocytic cells with a closely related pattern of surface receptors and monocyte- and macrophage-like behavior. ${ }^{20}$

Human umbilical vein endothelial cells were purchased (Clonetics) and cultured (for 2-4 passages) in low serum endothelial cell growth medium (PromoCell, Heidelberg, Germany).

\section{Protein Extraction}

Valve leaflets were inserted in a $2 \mathrm{~mol} / \mathrm{L}$ guanidinium chloride solution containing $0.01 \mathrm{~mol} / \mathrm{L} \mathrm{CaCl} 2,0.2 \%$ Triton X-100, and $0.05 \mathrm{~mol} / \mathrm{L}$ Tris$\mathrm{HCl}, \mathrm{pH} 7.5$ (3 mL/g wet weight) using a Polytron (Kinematica, Lucerne, Switzerland) as previously described. ${ }^{21}$ After centrifugation $(10,000 \mathrm{~g}$, 25 minutes, $48^{\circ} \mathrm{C}$ ), supernatants were dialyzed at $48^{\circ} \mathrm{C}$ for 48 hours (Spectrapor membrane 6-8000 MWC; Spectrum Laboratories, Inc, Rancho Dominguez, Calif). The dialyzed extracts were centrifuged $(10,000 g$, 25 minutes, $48^{\circ} \mathrm{C}$ ), and protein concentration was determined by the Bradford assay (Bio-Rad, Hercules, Calif). ${ }^{22}$

\section{Western Blot}

Western blot analysis was performed as previously described ${ }^{18}$ on aortic valve tissues collected from patients undergoing aortic valve replacement for aortic stenosis $(n=4)$ or heart transplantation for ischemic cardiomyopathy $(n=4)$. After extraction and homogenization, identical protein concentrations of each sample were loaded per lane on $10 \%$ gels and subjected to sodium dodecylsulfate polyacrylamide gel electrophoresis. Proteins were blotted and probed with mouse monoclonal antibodies anti-human EMMPRIN (HIM6, $10 \mu \mathrm{g} / \mathrm{mL}$; Becton Dickinson, Franklin Lakes, NJ), anti-human MT1-MMP (clone 114-1F2, $10 \mu \mathrm{g} / \mathrm{mL}$; Oncogene, Cambridge, Mass), anti-human MMP-9 (polyclonal, $10 \mu \mathrm{g} / \mathrm{mL}$; Millipore, Billerica, Mass), anti-human MMP-2 (clone 42-5D1, $10 \mu \mathrm{g} / \mathrm{mL}$; Calbiochem, Darmstadt, Germany), or anti-human $\beta$-actin (AC 15, $640 \mathrm{ng} / \mathrm{mL}$; SigmaAldrich, Munich, Germany). After they were washed, blots were incubated with biotinylated secondary antimouse antibody $(200 \mathrm{ng} / \mathrm{mL}$; Santa Cruz Biotechnology, Santa Cruz, Calif), and chemiluminescence staining was performed by Western Blotting Luminol Reagent (Santa Cruz Biotechnology), according to the manufacturer's instructions.

\section{Immunohistochemistry}

Formalin-fixed and paraffin-embedded tissue was cut in $2-\mu \mathrm{m}$ sections. After antigen retrieval (cooking with citrate buffer at $\mathrm{pH}$ 6) and blocking with $5 \%$ goat serum, slides were incubated with primary antibody solution for 1 hour at room temperature. The following antibodies and concentrations were used: CD68 (phosphoglucomutase-1, 1:50; DAKO, Glostrup, Denmark), EMMPRIN (1:200; abcam, Cambridge, Mass), MT1-MMP (1:200; abcam), MMP-2 (1:100; abcam), and MMP-9 (1:100; abcam). Detection was carried out using the DAKO Real Detection kit following the manufacturer's protocol. For double immunofluorescence, Cy5-labeled goat anti-rabbit (Invitrogen, Karlsruhe, Germany) and fluorescein isothiocyanate-labeled goat anti mouse immunoglobulin-G (Invitrogen) were used.

\section{In Situ Gelatin Zymography}

In situ zymography was performed, as previously described, ${ }^{23}$ by incubating cryosections $(7 \mu \mathrm{m})$ with $40 \mu \mathrm{g} / \mathrm{mL}$ fluorescein-conjugated gelatin (Invitrogen) in $50 \mathrm{mmol} / \mathrm{L}$ Tris- $\mathrm{HCl} \mathrm{pH} \mathrm{7.5,} 10 \mathrm{mmol} / \mathrm{L} \mathrm{CaCl}_{2}, 150 \mathrm{mmol} / \mathrm{L}$ $\mathrm{NaCl}$, and 0.05 Triton X-100 (Sigma-Aldrich) for 24 hours at $37^{\circ} \mathrm{C}$. Sections were washed 3 times with phosphate-buffered saline and mounted with Vectashield (BIOZOL, Eching, Germany). Gelatinase activity was visualized using fluorescent microscopy on an Olympus BX-50 microscope (Center Valley, Pa). Cells were counterstained using Hoechst 33342 (Invitrogen). Specificity of gelatinolytic activity was confirmed by incubating sections in the presence of EDTA/EGTA $(20 \mu \mathrm{M})$ or the MMP inhibitor GM6001 (Calbiochem) $(1 \mu \mathrm{mol} / \mathrm{L})$. 


\section{Transmigration Assays}

As an artificial valve model, a modified transmigration assay was performed using the Cell Invasion Assay Kit (Millipore) as previously described. ${ }^{24}$ In an invasion chamber, consisting of a 24-well tissue culture plate with 12 cell culture inserts, a thin layer of Matrigel (ECMatrix, Millipore) was dried over the inserts containing an 8- $\mu \mathrm{m}$ pore size polycarbonate membrane. The Matrigel layer occludes the membrane pores. Endothelial cells (human umbilical vein endothelial cells, PromoCell) were placed over the Matrigel in a confluent manner. Afterward, human monocytic cells (MonoMac6 cells, $2.5 \times 10^{5} / \mathrm{mL}$ ) were placed into the upper compartment of the transwells, either receiving control small interfering (si)RNA or pretreated with EMMPRIN-silencing siRNA (Qiagen, Hilden, Germany; EMMPRIN 2, target sequence GAC GGC CAT GCT GGT CTG CAA [dXdY-overhang]). Medium containing fetal bovine serum was introduced into the lower compartment beneath the filter. Subsequently, monocytic cells were allowed to migrate through a layer of ECM, covered by endothelial cells. After 48 hours, migrated MonoMac6 cells were harvested from the lower surface of the membrane and subsequently stained. Quantification was performed by colorimetric reading of optical density at $560 \mathrm{~nm}$.

\section{Statistical Analysis}

Results with normally distributed continuous variables were reported as mean \pm standard deviation and analyzed by the Student unpaired $t$ test. Statistical analysis for in situ zymography fluorescence intensity was performed using 1-way analysis of variance with post hoc Bonferroni multiple comparisons procedure.

\section{RESULTS}

\section{Immunohistochemistry}

Increased activity of MMPs has been demonstrated in calcific aortic stenosis. ${ }^{4}$ EMMPRIN has been established as a central activator of MMPs, increasing the secretion of pro-MMPs and enhancing the expression of membranetype MMPs, such as MT1-MMP on the cell surface. MT1-MMP facilitates migration of monocytes through activated endothelial cells. ${ }^{25}$

To examine if EMMPRIN and MT1-MMP are expressed in calcific aortic stenosis, immunohistochemical analysis was performed on 12 stenotic valves containing degenerative calcific tissue and on 2 noncalcified control valves. All 12 stenotic valves stained positive for EMMPRIN, MT1-MMP, MMP-9, MMP-2, and the macrophage marker CD68, whereas neither of the controls did so for any marker. We used breast cancer as a control for positive EMMPRIN and MT1-MMP staining. Staining of MT1-MMP, EMMPRIN, MMP-2, and MMP-9 was highest in regions of inflammation, in the border zone (leading edge) to calcifications. Measurement of positive staining area to the total area demonstrated a value of $5 \%( \pm 2 \%)$. Macrophages staining for EMMPRIN and MT1-MMP were mainly localized within the layer of fibrosa (Figure 1).

\section{Double Immunofluorescence Staining}

To confirm the immunohistologic results to localize EMMPRIN and MT1-MMP, double immunofluorescence staining was performed for EMMPRIN, MT1-MMP, and

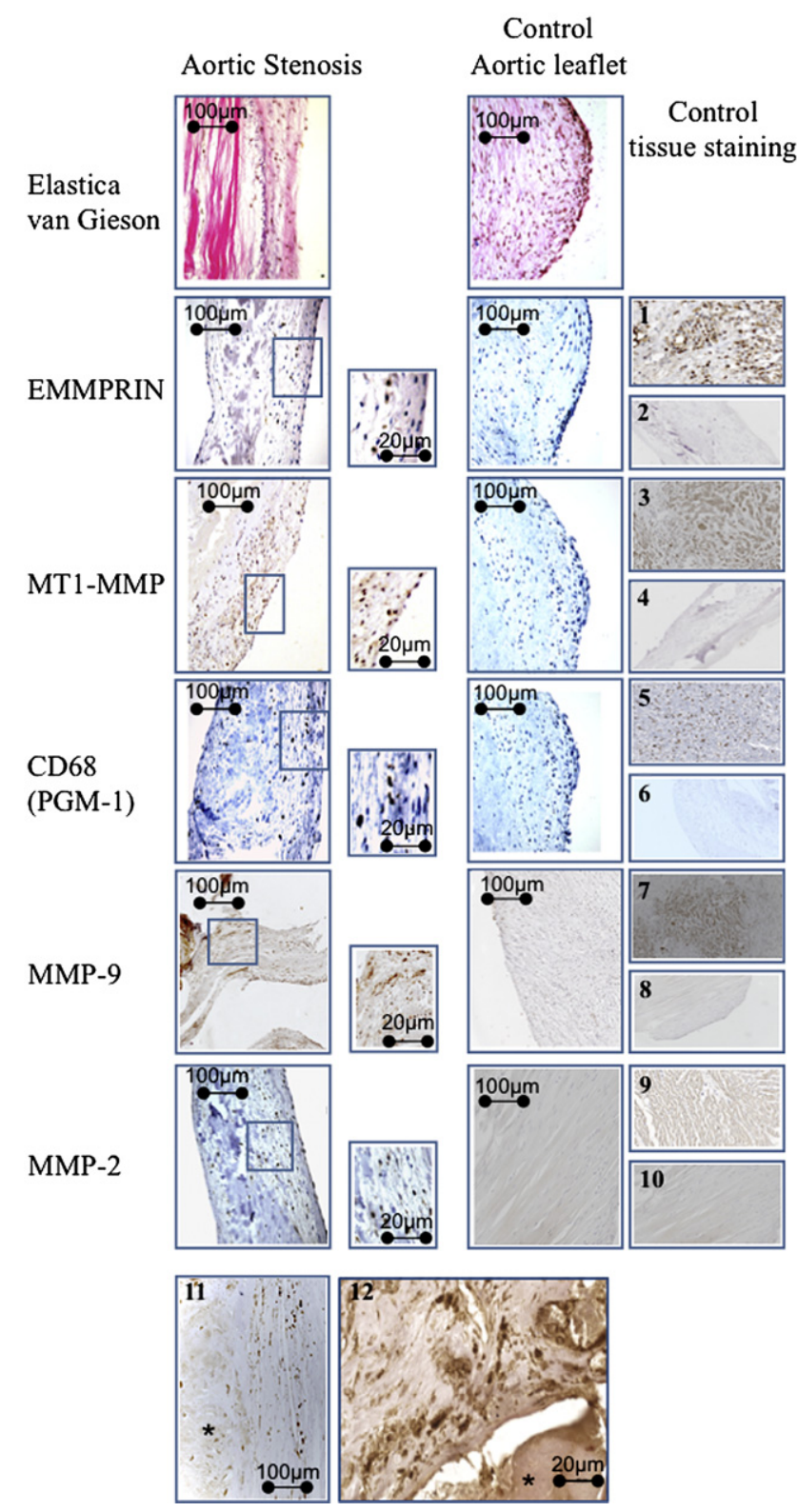

FIGURE 1. EMMRIN and MT1-MMP in aortic stenosis. Representative degenerative aortic valve tissue (left) and control aortic leaflet (center) are shown. In arteriosclerotic valves, Elastica van Gieson staining demonstrates a characteristic lesion with dense collagen (staining red), whereas control patients exhibit loose connective tissue with subendothelial localization. There is a clearly visible increase in CD68-positive macrophages and cells demonstrating EMMPRIN and MT1-MMP compared with control leaflets. In arteriosclerotic valves, the majority of cells demonstrate increased protein levels of MMP-2 and MMP-9 when compared with control tissue. Right column demonstrates control stainings (positive controls: breast carcinoma for EMMRIN (1), MT1-MMP (3), MMP-9 (7), and MMP-2 (9), and lymph node for CD68 (5); subclass-matched irrelevant immunoglobulin-G served as negative controls: $(2,4,6,8,10)$. EMMPRIN-staining cells accumulate in the border zone to calcification $(*)(11)$, which is stained by alizarin red (12), demonstrating that the changes in EMMPRIN activity occur in the calcific/ per-calcific regions of the valve. MTI-MMP, Membrane type 1-matrix metalloproteinase; $P G M$, phosphoglucomutase. 

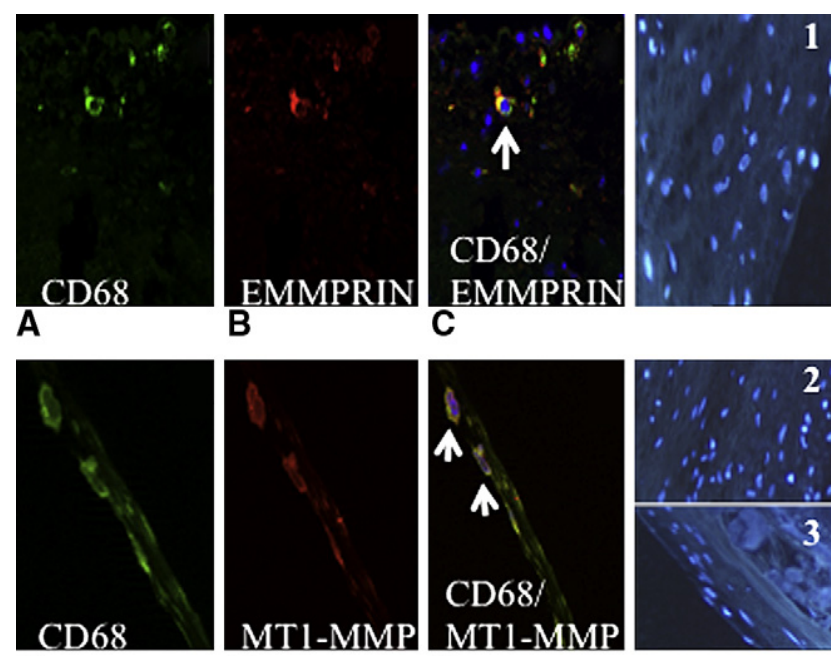

$$
\text { D }
$$
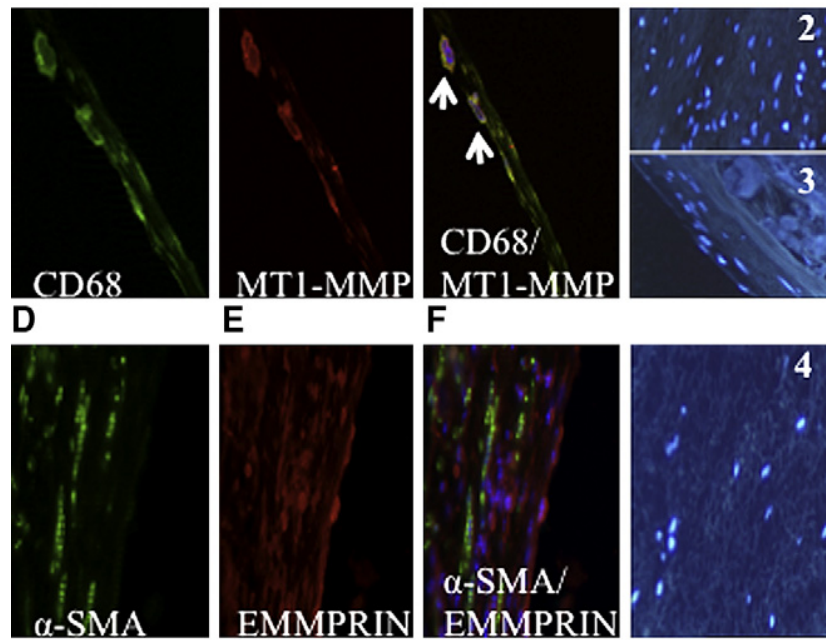

G E $F$
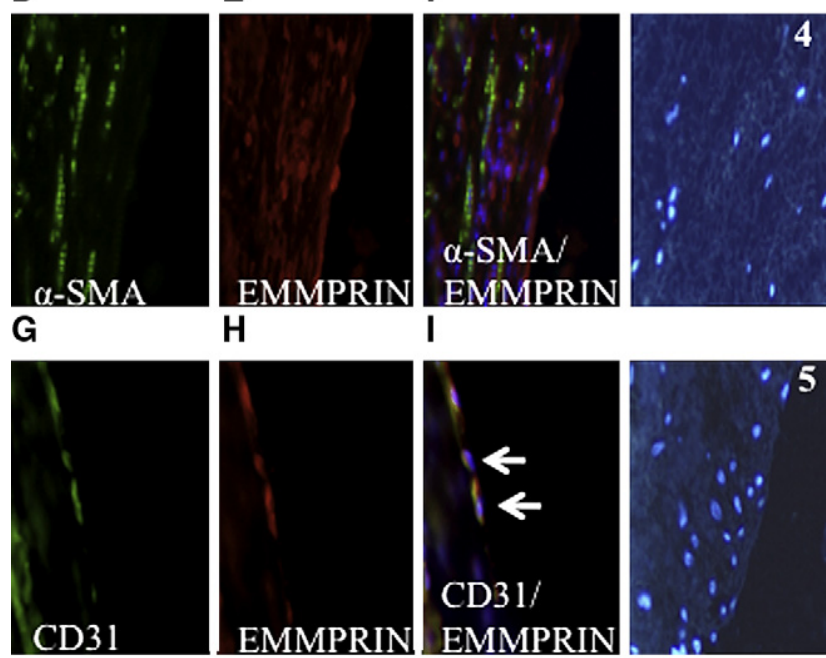
H I
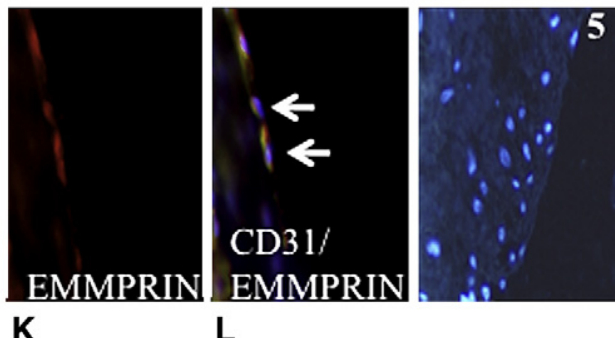

J

FIGURE 2. Double immunofluorescence for EMMPRIN and MT1-MMP in aortic valve tissue. Green fluorescence (fluorescein isothiocyanate) denotes tissue macrophage staining positive for CD68 (A, D), alpha smooth muscle actin (G), and CD31 (J). Red fluorescence (Cy5) demonstrates cells positive for EMMPRIN (B, H, K) and MT1-MMP (E). Double immunofluorescence shows that EMMPRIN and MT1-MMP are co-expressed with CD68 in altered aortic valves (C, F, arrows). This proves that infiltrating macrophages in sclerotic valves express MT1-MMP and EMMPRIN. Blue dye (Hoechst, Invitrogen, Karlsruhe, Germany) identifies nucleated cells. EMMPRIN is not co-expressed with ( $\alpha$-SMA) (I), and EMMPRIN colocalizes with CD31 (L, arrows). Right column represents negative control stainings for EMMPRIN (1), CD68 (2), MT1-MMP (3), $\alpha$-SMA (4), and CD31 (5). SMA, Smooth muscle actin.

CD68. CD68-positive macrophages co-stained for EMMPRIN and MT1-MMP, showing that in the degenerative aortic valve, activated macrophages express both EMMPRIN and MT1-MMP on the cell surface. EMMPRIN co-stained with CD31, a marker of endothelial cells, but did not co-stain with $\alpha$-smooth muscle actin, a marker of activated valve interstitial cells (Figure 2).
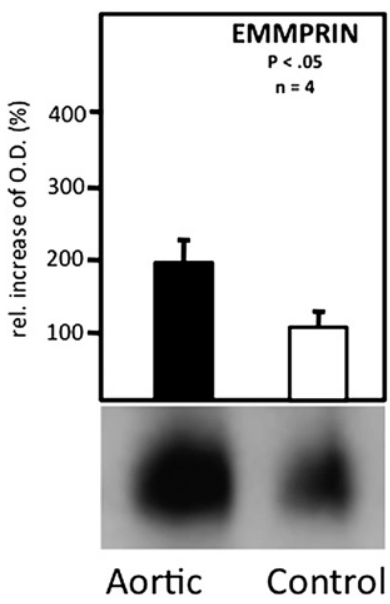

A stenosis

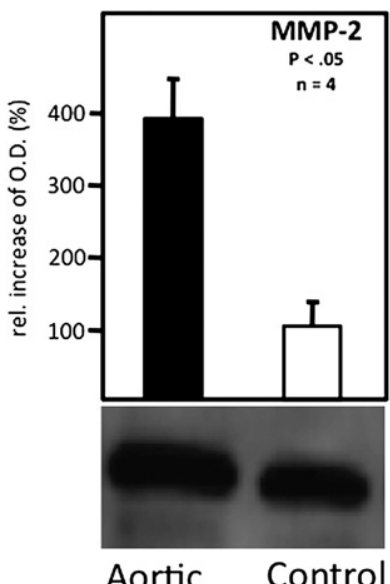

\section{C stenosis}
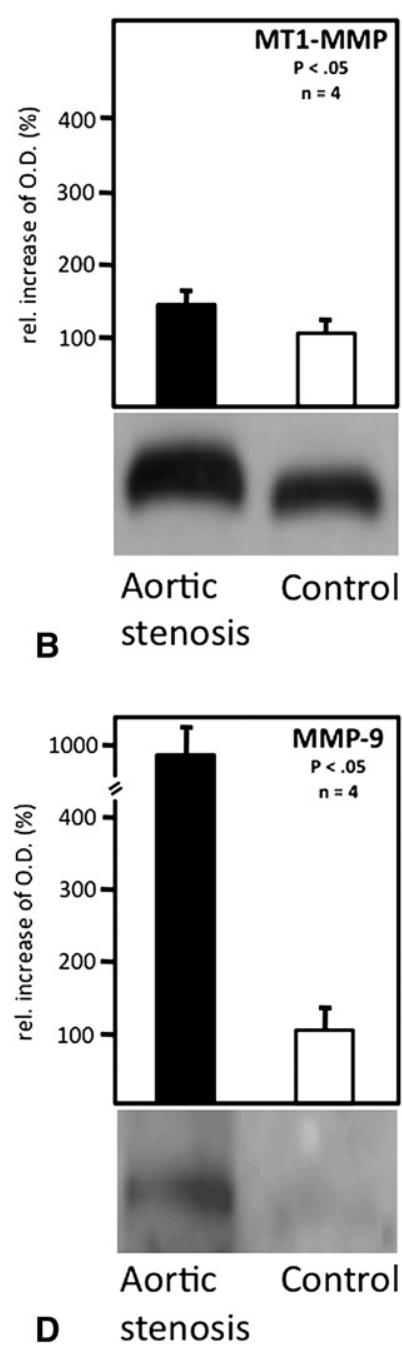

FIGURE 3. Western blot immunoreactivity of EMMPRIN, MT1-MMP, MMP-2, and MMP-9. Representative Western blot analysis of aortic valve tissue demonstrates increased EMMPRIN (A), MT1-MMP (B), MMP-2 (C), and MMP-9 (D) protein level (to statistically significant extent, $P<.05$ ) in aortic stenosis (left column) compared with control aortic valve tissue (right column). B-actin serves as control protein. MMP, Matrix metalloproteinase.

\section{Biochemical Analysis}

These results prompted us to investigate the protein expression of EMMPRIN, MT1-MMP, MMP-9, and MMP-2. Western blot immunoreactivity of EMMPRIN, MT1-MMP, MMP-9, and MMP-2 was detected in aortic stenosis samples at a greater staining intensity than in control aortic valve tissues $(P<.05)$. $\beta$-actin was used as control protein (Figure 3).

\section{In Situ Gelatin Zymography}

In situ gelatin zymography was performed to determine whether increases in EMMPRIN or MT1-MMP, as demonstrated by immunohistochemistry and Western blot, are 


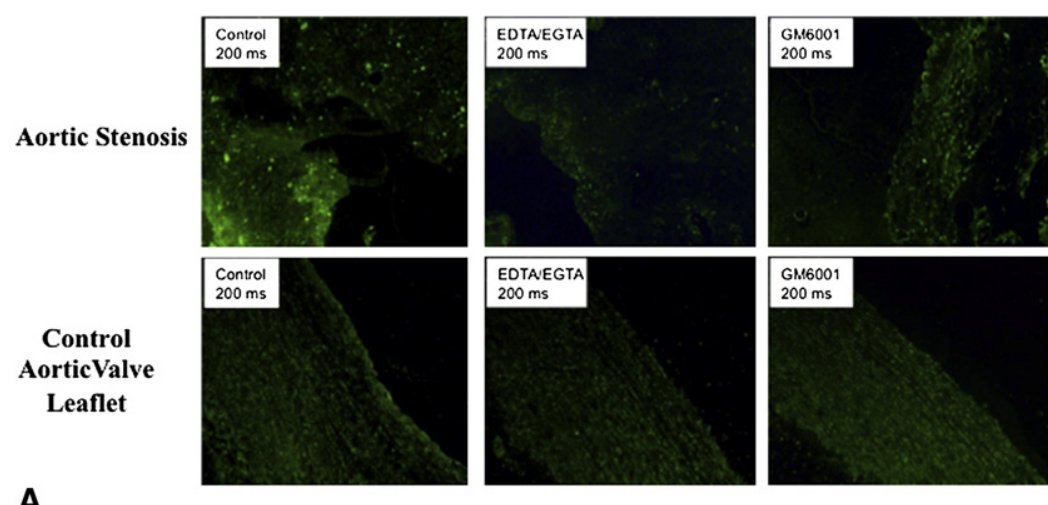

A

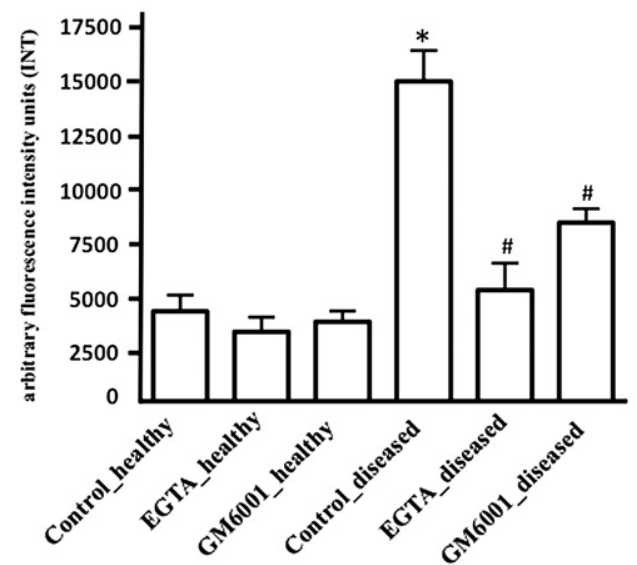

${ }^{*} \mathrm{p}<0.05$ vs. healthy control

$\# p<0.05$ vs. diseased control

B

FIGURE 4. A, B, In situ gelatin zymography. Representative zymographs (A) of stenotic aortic valve (upper) demonstrate basic gelatinolytic activity. This activity is dampened by the MMP inhibitor GM6001. In control aortic valves (lower) gelatinolytic activity is weak when compared with stenotic aortic valves. Gelatinolytic specificity of activity is confirmed by incubating samples in the presence of EGTA. Exposure time: $200 \mathrm{~ms}$. Intensity of the in situ zymography fluorescence was quantified using Quantity One software (Version 4.4.1) from BioRad (Hercules, CA). Data are shown (B) as mean \pm standard error of the mean of arbitrary fluorescence intensity $(n=4)$. Basic gelatinolytic activity was significantly increased in stenotic aortic valves (controldiseased) compared with non-diseased aortic valves (control-healthy) $\left({ }^{*} P<.05\right)$. Moreover, gelatinolytic activity was significantly dampened by the MMP inhibitor GM6001 when compared with the basic gelatinolytic activity of the stenotic aortic valves $\left({ }^{\#} P<.05\right)$. OD, Optical density; siRNA, small interfering RNA; MMP, matrix metalloproteinase.

associated with local increases in MMP activity. In stenotic aortic valve specimens, the basic gelatinolytic activity is increased when compared with control aortic valves $(P<.05$, $\mathrm{n}=4)$. This activity is dampened by the MMP inhibitor GM6001 $(P<.05, \mathrm{n}=4)$ (Figure 4).

\section{Transmigration Assays}

Enhanced protein expression of EMMPRIN and MT1-MMP on monocytic cells in aortic stenosis led us to investigate whether EMMPRIN was capable of affecting transmigration of circulating monocytes into the subendothelial space to foster the inflammatory process. For this, an artificial valve model was used, composed of ECM covered by endothelial cells (human umbilical vein endothelial cells). The EMMPRIN gene in human monocytic MonoMac cells was silenced with EMMPRIN-silencing siRNA for 48 hours, and cell transmigration through the reconstructed artificial valve surface was studied. EMMPRINsilencing siRNA significantly reduced transmigration of monocytic cells compared with pretreatment with control siRNA $(P<.05)$. A similar effect was demonstrated by pretreating monocytic cells with an MMP inhibitor (GM6001) compared with control peptide $(P<.05)$. For transmigration of monocytic cells, EMMPRIN-specific siRNA had no further effect when added to MMP inhibitor (GM6001) (Figure 5).

\section{DISCUSSION}

MMPs are involved in both the pathologic remodeling of the left ventricle in patients with aortic valve stenosis ${ }^{26}$ and the stenotic aortic valve tissue itself. ${ }^{6}$ Tumor cell-derived EMMPRIN has been shown to regulate the activity of 


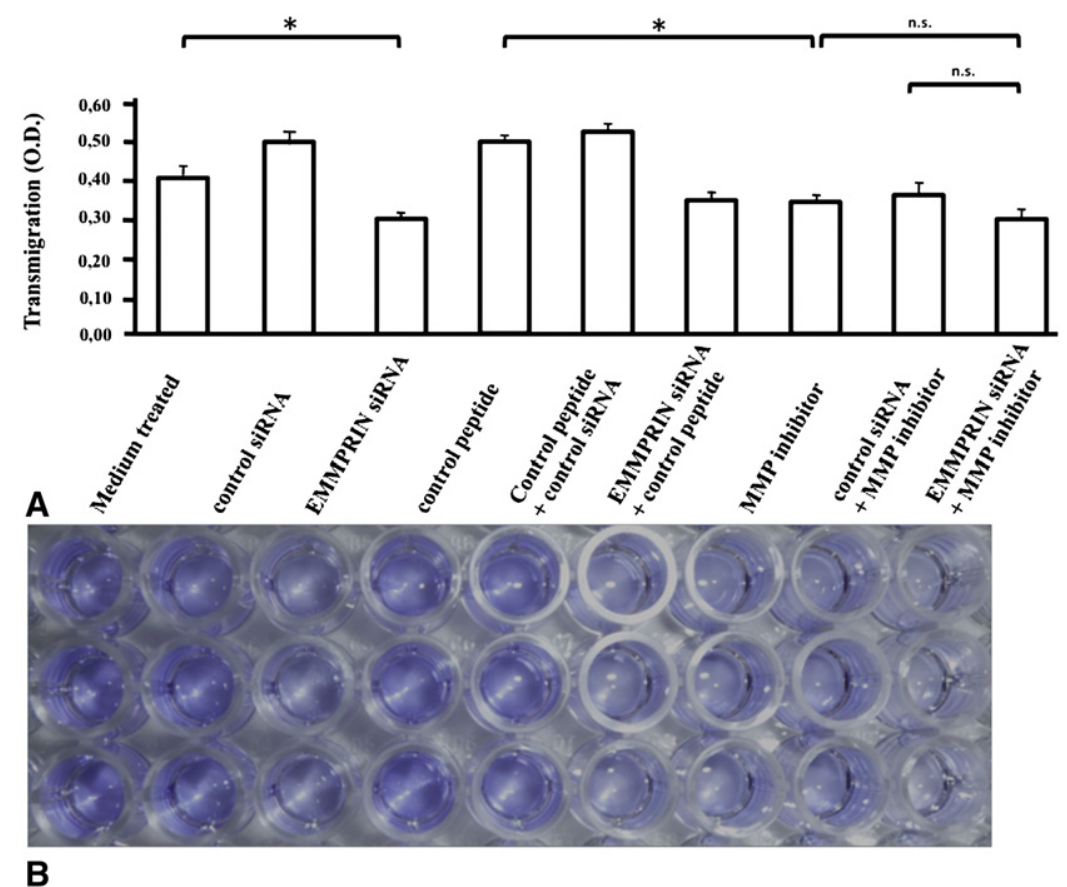

FIGURE 5. A, B, Transmigration through an artificial valve surface quantitative analysis (A) and representative images from the transmigration studies (B). Human monocytic MonoMac6 cells were treated with control siRNA or EMMPRIN-silencing siRNA for 48 hours, and their transmigration through the composite of endothelial cell layer and Matrigel (Millipore, Billerica, Mass) was determined. EMMPRIN-silencing siRNA significantly reduced transmigration of monocytic cells compared with pretreatment with control siRNA $(* P<.05)$. A similar effect was demonstrated by pretreating monocytic cells with the MMP inhibitor GM6001 (Calbiochem, Darmstadt, Germany) compared with control peptide $(* P<.05)$. EMMPRIN-specific siRNA had no additive effect to MMP inhibition for transmigration of monocytic cells $(P=.0621)$. NS, Not significant; $O D$, optical density.

MMPs in surrounding fibroblasts. ${ }^{17}$ Among these MMPs, the membrane type 1 (MT1)-MMP has been regarded as a major activator of soluble (pro-)MMPs, especially of pro-MMP2. ${ }^{27}$

The current study shows that EMMPRIN and MT1-MMP are increased in degenerative stenotic aortic valves compared with normal control aortic valves. EMMPRIN localizes to monocytes and endothelial cells in aortic valve tissue and provides evidence that inhibition of the EMMPRIN gene may reduce migration of monocytes into the valve.

Immunohistochemistry and Western blot analysis confirmed previous data demonstrating an increase in MMP-2 and MMP-9 protein in calcified stenotic aortic valves, reflecting the presence of inflammation. Some, but not all, ${ }^{28}$ studies have indicated a potential involvement of MMP-2 in the development of aortic valve disease. MMP-2 was previously associated with tenascin-C, an ECM glycoprotein found in developing bone and atherosclerotic plaque, in calcific aortic valve stenosis. Moreover, the mRNA expression and gelatinolytic activity of MMP-2 were up-regulated when aortic valve interstitial cells were cultivated on collagen supplemented with tenascin-C, thus supporting the hypothesis that MMP-2 may be associated with progression of calcification. ${ }^{29}$ Immunohistochemistry demonstrated EMMPRIN in calcific degenerative specimens, a result we confirmed using Western blotting. In histologic studies,
EMMPRIN was found in close proximity to CD68-positive tissue macrophages and CD31-positive endothelial cells. Double immune fluorescence staining co-localized EMMPRIN with CD68 and CD31, suggesting that monocytes entering the aortic valve and endothelial cells contain EMMPRIN. Taken together, these findings suggest that migration of monocytes into aortic valve tissue may be affected by an EMMPRIN-based interaction of monocytes and endothelial cells.

On the basis of observations in tumors ${ }^{30}$ and cardiovascular cells, ${ }^{18}$ EMMPRIN may induce proteolysis by activating MMPs in calcific aortic valve stenosis and thus may influence the pathologic remodeling process. We are not aware of prior data on EMMPRIN-mediated proteolytic effects in calcific aortic valve stenosis, so our results represent the first described association between EMMPRIN/MT1MMP and aortic valve stenosis. Our results are not sufficient to prove that EMMPRIN plays a causal role in the development of aortic stenosis in vivo. To draw such a conclusion, it would be necessary to investigate its role in a good animal model of aortic stenosis, something that has raised questions with regard to the extrapolation to humans. ${ }^{31}$ Moreover, in the mouse, knocking out the EMMPRIN gene has usually proved lethal. ${ }^{32}$ Thus, a combination of these approaches is clearly beyond the scope of our investigation at the present time. An indication of the potential functional 
relevance of our findings may be gained from our finding that EMMPRIN induces migration of macrophages across a biological barrier resembling the endothelial cells lining the aortic valve. Other EMMPRIN-mediated effects, such as an increase in the production of MMP-2 and MMP-9 in the aortic valve, will also require evaluation in appropriate models, as will the correlation of EMMPRIN activity with the extent of calcification and the clinical outcome. Other inflammatory sequelae may be caused by MMPmediated cleavage products, as has been demonstrated for MMP- $2^{33}$ and MMP- $9^{34}$ in other disease entities, although no studies exist regarding this mechanism in aortic stenosis.

Because protease activation in monocytes is linked to nuclear factor kappa-B ( $\mathrm{NF}_{\kappa}$-B) signaling, proinflammatory downstream effects in aortic valve stenosis may involve the NF $\kappa$-B signaling cascade. We previously demonstrated that cell-cell communication via EMMPRIN activates $\mathrm{NF} \kappa-\mathrm{B}$ via the $\mathrm{I} \kappa \mathrm{B} \alpha$ complex. In monocytes, $\mathrm{NF} \kappa-\mathrm{B}$ is known to activate the synthesis of proinflammatory cytokines, such as interleukin-6 and TNF- $\alpha$. Pretreatment of monocytes with an IKK inhibitor or EMMPRIN-specific siRNA sharply hindered EMMPRIN-mediated cytokine induction $^{35}$; however, the implications of these data in the setting of aortic valve stenosis remain to be clarified.

TNF- $\alpha$ may promote an osteoblast-like phenotype in human aortic valve myofibroblasts and thus at least partially regulate the process of aortic valve calcification. ${ }^{36}$ It is possible that EMMPRIN-overexpressing monocytes, by secretion of TNF- $\alpha$, are also capable of promoting an osteoblast-like phenotype in aortic valve myofibroblasts and thus contribute to calcification, but this is speculative at the present time.

Little is known about EMMPRIN and MT1-MMP interaction in aortic valve tissues. Because constitutive EMMPRIN knockout models have proved lethal, ${ }^{32}$ developing conditional knockout models may clarify EMMPRIN's role in the course of calcific aortic valve stenosis. Future studies should focus on specific effects of EMMPRIN and MT1-MMP on inflammation and calcification in aortic valve stenosis and identify their particular contribution, possibly by elaborating novel ligands for intracellular activation cascades.

\section{Limitation}

Sample size is a major limiting factor for this study. Nonetheless, we performed statistical analysis for the Western blot results demonstrating significantly increased protein levels of EMMPRIN and MT1-MMP in stenotic aortic valves compared with control aortic valves.

\section{CONCLUSIONS}

The present study demonstrated both EMMPRIN and MT1-MMP on macrophages in the stenotic aortic valve, suggesting a pathway by which macrophages may induce proteolysis-mediated migration of macrophages into developing aortic valve stenosis. Silencing the EMMPRIN gene using siRNA halted monocytic transmigration in an artificial valve model. These findings may assist in the development of more nuanced anti-inflammatory strategies inhibiting calcific and eventually degenerative sequelae in aortic valve stenosis.

The authors thank Monika Hölderle and Renate Hegenloh for expert technical assistance.

\section{References}

1. Iung B, Baron G, Butchart EG, Delahaye F, Gohlke-Barwolf C, Levang OW, et al. A prospective survey of patients with valvular heart disease in Europe: The Euro Heart Survey on Valvular Heart Disease. Eur Heart J. 2003;24: 1231-43.

2. Babaliaros V, Block P. State of the art percutaneous intervention for the treatment of valvular heart disease: a review of the current technologies and ongoing research in the field of percutaneous valve replacement and repair. Cardiology. 2007;107:87-96.

3. Rosenhek R, Baumgartner H. Aortic sclerosis, aortic stenosis and lipid-lowering therapy. Expert Rev Cardiovasc Ther. 2008;6:385-90.

4. Helske S, Kupari M, Lindstedt KA, Kovanen PT. Aortic valve stenosis: an active atheroinflammatory process. Curr Opin Lipidol. 2007;18:483-91.

5. Miller JD, Chu Y, Brooks RM, Richenbacher WE, Peña-Silva R, Heistad DD. Dysregulation of antioxidant mechanisms contributes to increased oxidative stress in calcific aortic valvular stenosis in humans. J Am Coll Cardiol. 2008; 52:843-50.

6. Koullias GJ, Korkolis DP, Ravichandran P, Psyrri A, Hatzaras I, Elefteriades JA. Tissue microarray detection of matrix metalloproteinases, in diseased tricuspid and bicuspid aortic valves with or without pathology of the ascending aorta. Eur J Cardiothorac Surg. 2004;26:1098-103.

7. Aikawa E, Aikawa M, Libby P, Figueiredo JL, Rusanescu G, Iwamoto Y, et al. Arterial and aortic valve calcification abolished by elastolytic cathepsin S deficiency in chronic renal disease. Circulation. 2009;119:1785-94.

8. Aikawa E, Nahrendorf M, Figueiredo JL, Swirski FK, Shtatland T, Kohler RH, et al. Osteogenesis associates with inflammation in early-stage atherosclerosis evaluated by molecular imaging in vivo. Circulation. 2007;116:2841-50.

9. Aikawa E, Nahrendorf M, Sosnovik D, Lok VM, Jaffer FA, Aikawa M, et al. Multimodality molecular imaging identifies proteolytic and osteogenic activities in early aortic valve disease. Circulation. 2007;115:377-86.

10. Rajamannan NM, Subramaniam M, Caira F, Stock SR, Spelsberg TC. Atorvastatin inhibits hypercholesterolemia-induced calcification in the aortic valves via the Lrp5 receptor pathway. Circulation. 2005;112:I229-34.

11. Inoue I, Goto S, Awata T, Mastunaga T, Kawai S, Nakijama T, et al. Lipophilic HMG-CoA reductase inhibitor has an anti-inflammatory effect: reduction of MRNA levels for interleukin-1beta, interleukin-6, cyclooxygenase-2, and p22phox by regulation of peroxisome proliferator-activated receptor alpha (PPARalpha) in primary endothelial cells. Life Sci. 2000;67:863-76.

12. Rosenson RS, Tangney CC, Casey LC. Inhibition of proinflammatory cytokine production by pravastatin. Lancet. 1999;353:983-4.

13. Laufs U, Marra D, Node K, Liao JK. 3-Hydroxy-3-methylglutaryl-CoA reductase inhibitors attenuate vascular smooth muscle proliferation by preventing rho GTPase-induced down-regulation of p27(Kip1). J Biol Chem. 1999;274: 21926-31

14. Kizu A, Shioi A, Jono S, Koyama H, Okuno Y, Nishizawa Y. Statins inhibit in vitro calcification of human vascular smooth muscle cells induced by inflammatory mediators. J Cell Biochem. 2004;93:1011-9.

15. Chan KL, Teo K, Dumesnil JG, Ni A, Tam J, ASTRONOMER Investigators Effect of Lipid lowering with rosuvastatin on progression of aortic stenosis: results of the aortic stenosis progression observation: measuring effects of rosuvastatin (ASTRONOMER) trial. Circulation. 2010;121:306-14.

16. Newby AC. Metalloproteinase expression in monocytes and macrophages and it relationship to atherosclerotic plaque instability. Arterioscler Thromb Vasc Biol. 2008;28:2108-14.

17. Biswas C, Zhang Y, DeCastro R, Guo H, Nakamura T, Kataoka H, et al. The human tumor cell-derived collagenase stimulatory factor (renamed EMMPRIN) is a member of the immunoglobulin superfamily. Cancer Res. 1995;55:434-9. 
18. Schmidt R, Bültmann A, Ungerer M, Joghetaei N, Bülbül Ö, Thieme S, et al. EMMPRIN regulates MMP activity in cardiovascular cells. Implications in acute myocardial infarction. Circulation. 2006;113:834-41.

19. Schmidt R, Redecke V, Breitfeld Y, Wantia N, Miethke T, Massberg S, et al. EMMPRIN (CD 147) is a central activator of extracellular matrix degradation by Chlamydia pneumoniae-infected monocytes. Implications for plaque rupture. Thromb Haemost. 2006;95:151-8.

20. May AE, Redecke V, Grüner S, Schmidt R, Massberg S, Miethke T, et al. Recruitment of Chlamydia pneumoniae-infected macrophages to the carotid artery wall in noninfected, nonatherosclerotic mice. Arterioscler Thromb Vasc Biol. 2003; 23:789-94

21. Badier-Commander C, Verbeuren T, Lebard C, Michel JB, Jacob MP. Increased TIMP/MMP ratio in varicose veins: a possible explanation for extracellular matrix accumulation. J Pathol. 2000;192:105-12.

22. Bradford MM. A rapid and sensitive method for the quantitation of microgram quantities of protein utilizing the principle of protein-dye binding. Anal Biochem. 1976;72:248-54.

23. Masson V, de la Ballina LR, Munaut C, Wielockx B, Jost M, Maillard C, et al. Contribution of host MMP-2 and MMP-9 to promote tumor vascularization and invasion of malignant keratinocytes. FASEB J. 2005; 19:234-6.

24. Klouche M, May AE, Hemmes M, Messner M, Kanse SM, Preissner KT, et al. Enzymatically modified, nonoxidized LDL induces selective adhesion and transmigration of monocytes and T-lymphocytes through human endothelial cell monolayers. Arterioscler Thromb Vasc Biol. 1999;19:784-9.

25. Sithu SD, English WR, Olson P, Krubasik D, Baker AH, Murphy G, et al. Membrane-type 1-matrix metalloproteinase regulates intracellular adhesion molecule1 (ICAM-1)-mediated monocyte transmigration. J Biol Chem. 2007;282:25010-9.

26. Fielitz J, Leuschner M, Zurbrügg HR, Hannack B, Pregla R, Hetzer R, et al. Regulation of matrix metalloproteinases and their inhibitors in the left ventricular myocardium of patients with aortic stenosis. J Mol Med. 2004;82:809-20.

27. Sounni NE, Devy L, Hajitou A, Frankenne F, Munaut C, Gilles C, et al. MT1-MMP expression promotes tumor growth and angiogenesis through an up-regulation of vascular endothelial growth factor expression. FASEB J. 2002; 16:555-64.
28. Parolari A, Loardi C, Mussoni L, Cavallotti L, Camera M, Biglioli P, et al. Nonrheumatic calcific aortic stenosis: an overview from basic science to pharmacological prevention. Eur J Cardiothorac Surg. 2009;35:493-504.

29. Jian B, Jones PL, Li Q, Mohler ER 3rd, Schoen FJ, Levy RJ. Matrix metalloproteinase-2 is associated with tenascin-C in calcific aortic stenosis. Am J Pathol. 2001;159:321-7.

30. Sameshima T, Nabeshima K, Toole BP, Yokogami K, Okada Y, Goya T, et al. Glioma cell extracellular matrix metalloproteinase inducer (EMMPRIN) (CD147) stimulates production of membrane-type matrix metalloproteinases and activated gelatinase A in co-cultures with brain-derived fibroblasts. Cancer Lett. 2000;157: 177-84.

31. Hekimian G, Passefort S, Louedec L, Houard X, Jacob MP, Vahanian A, et al. High-cholesterol + vitamin D2 regimen: a questionable in-vivo experimental model of aortic valve stenosis. J Heart Valve Dis. 2009;18:152-8.

32. Igakura T, Kadomatsu K, Kaname T, Muramatsu H, Fan QW, Miyauchi T, et al. A null mutation in basigin, an immunoglobulin superfamily member, indicates its important roles in peri-implantation development and spermatogenesis. Dev Biol. 1998;194:152-65.

33. Reinboldt S, Wenzel F, Rauch BH, Hohlfeld T, Grandoch M, Fischer JW, et al. Preliminary evidence for a matrix metalloproteinase-2 (MMP-2)-dependent shedding of soluble CD40 ligand (sCD40L) from activated platelets. Platelets. 2009;20:441-4.

34. Menchén L, Marín-Jiménez I, Arias-Salgado EG, Fontela T, HernándezSampelayo P, Rodríguez MC, et al. Matrix metalloproteinase 9 is involved in Crohn's disease-associated platelet hyperactivation through the release of soluble CD40 ligand. Gut. 2009;58:920-8.

35. Schmidt R, Bültmann A, Fischel S, Gillitzer A, Cullen P, Walch A, et al. Extracellular matrix metalloproteinase inducer (CD147) is a novel receptor on platelets, activates platelets, and augments nuclear factor kappaB-dependent inflammation in monocytes. Circ Res. 2008;102:302-9.

36. Kaden JJ, Kiliç R, Sarikoç A, Hagl S, Lang S, Hoffmann U, et al. Tumor necrosis factor alpha promotes an osteoblast-like phenotype in human aortic valve myofibroblasts: a potential regulatory mechanism of valvular calcification. Int J Mol Med. 2005;16:869-72. 